\title{
Effects of uncertainty in rock-physics models on reservoir parameter estimation using marine seismic AVA and CSEM data
}

\author{
Jinsong Chen*, Lawrence Berkeley National Laboratory, and Thomas Dickens, ExxonMobil Upstream \\ Research Company
}

\section{Summary}

This study investigates the effects of uncertainty in rockphysics models on estimates of reservoir parameters from joint inversion of seismic AVA and CSEM data. The reservoir parameters are related to electrical resistivity using Archie's law, and to seismic velocity and density using the $\mathrm{Xu}$-White model. To account for errors in the rock-physics models, we use two methods to handle uncertainty: (1) the model outputs are random functions with modes or means given by the model predictions, and (2) the parameters of the models are themselves random variables. Using a stochastic framework and Markov Chain Monte Carlo methods, we obtain estimates of reservoir parameters as well as of the uncertainty in the estimates. Synthetic case studies show that uncertainties in both rock-physics models and their associated parameters can have significant effects on estimates of reservoir parameters. Our method provides a means of quantifying how the uncertainty in the estimated reservoir parameters increases with increasing uncertainty in the rock-physics model and in the model parameters. We find that in the example we present, the estimation of water saturation is relatively less affected than is the estimation of clay content and porosity.

\section{Introduction}

Rock-physics models are needed to estimate reservoir parameters from seismic AVA and CSEM data. In practice, model parameters are often derived from nearby well logs. First, an appropriate family of rock-physics models is chosen, such as the sand-clay models of Xu and White (1995) for seismic velocities and density, and Archie's law (Archie, 1942) for resistivity. Second, the parameters associated with the rock-physics models are estimated by fitting them to the selected well log data. Since the relationships between reservoir parameters and geophysical attributes are nonlinear and non-unique, the derived rock-physics models and their parameters are uncertain to some extent. Such uncertainty may significantly affect estimates of reservoir parameters from geophysical data.

Traditional methods for analyzing uncertainty in rock-physics models entail varying a small subset of the rock-physics model parameters while keeping others unchanged. Such methods are valid only when the parameters being investigated are uncorrelated with those being kept fixed. Since rock-physics parameters often depend upon each other, the methods have limited validity. Additionally, those methods typically analyze only the effects of uncertainty in the rock-physics model parameters (Type 2), but not the uncertainty in the rock-physics model outputs (Type 1).

An alternative for studying the effects of uncertainty in rockphysics models is to utilize a Bayesian framework to represent geophysical properties as random functions of reservoir parameters. In this method, the rock-physics models derived from borehole logs provide only reference values (e.g., means or modes) for the reservoir parameters being estimated. The actual values are realizations drawn from the estimated a posteriori probability density functions, conditioned on the uncertainty in the rock-physics model as well as on the input seismic AVA and CSEM data. An example of such an approach is given by Bacharach (2006), where sediment bulk and shear moduli and density are considered to be random functions of reservoir water saturation and porosity, and the unknown reservoir parameters are estimated jointly by conditioning on seismic AVA data. Bacharach investigates only the effect of uncertainty in the rock-physics models (Type 1), but not the effect of uncertainty in the associated model parameters (Type 2). In practice, both types of uncertainty exist, and they may affect the estimates of reservoir parameters differently.

In this study, we investigate the effects of uncertainty in rock-physics models on reservoir parameter estimation, caused by uncertainty in the rock-physics model (Type 1) and uncertainty in the model parameters (Type 2). We develop a stochastic method based on a layered reservoir model, similar to the one studied by Chen et al. (2007), with the addition of stochastic rock-physics models to account for their contribution to the uncertainty. We use Markov chain Monte Carlo (MCMC) methods to explore the joint a posteriori probability density functions of the inverted parameters.

\section{Method}

$\underline{\text { Stochastic rock-physics models }}$

We relate reservoir water saturation $S_{w}$ and porosity $\varphi$ to electrical resistivity using Archie's law (Archie, 1942):

$$
r=r_{b} S_{w}^{-m} \varphi^{-n}
$$

Here, $r$ denotes the electrical resistivity of a given reservoir layer and the model parameters $r_{b}, m$, and $n$ denote brine resistivity and model exponents of water saturation and porosity, respectively. Two types of uncertainty may exist in this equation. The first is the uncertainty associated with the model parameters $r_{b}, m$, and $n$ (Type 2). To account for such uncertainty, we consider those parameters to be random 


\section{Effects of uncertainty in rock-physics models}

variables with given distributions. The second type of uncertainty is that associated with the model itself (Type 1), such as the discrepancy between the measured resistivity and the predicted resistivity; i.e., Archie's Law may not be an appropriate model for some situations. Similarly, to account for the effect of this type of uncertainty, we consider predicted resistivity to be a random function of water saturation, porosity, and the model parameters, while using Archie's Law to calculate the mode of the distribution.

We use a Gamma distribution function with shape parameter $\alpha$ and scale parameter $\beta$ to describe the uncertainty associated with the model given in Equation 1. Let the vector $\boldsymbol{\theta}_{1}=\left(r_{b}, m, n\right)^{T}$, where $T$ denotes transpose. Let the mode of the Gamma distribution be equal to the resistivity predicted by Equation 1. Consequently, we obtain the conditional distribution function of resistivity given reservoir parameters $S_{w}$ and $\varphi$ and unknown model parameters $\boldsymbol{\theta}_{1}$, as follows:

$$
f\left(r \mid S_{w}, \varphi, \boldsymbol{\theta}_{1}\right)=\frac{r^{\alpha-1}}{\beta^{\alpha} \Gamma(\alpha)} \exp \left(-\frac{r}{\beta}\right) .
$$

We relate seismic P- and S-wave velocity and density to reservoir parameters using a clay-sand mixture model developed by $\mathrm{Xu}$ and White (1995). The main parameters associated with the $\mathrm{Xu}$-White model are the bulk and shear moduli and density of sand grains, clay, and fluid, and the pore aspect ratios of sand and clay. The reservoir parameters that affect seismic P- and S-wave velocity and density are water saturation, clay content, and porosity. We use the vector $\boldsymbol{\theta}_{2}$ to represent the entire set of model parameters, and consider the parameters to be random variables in order to model parameter uncertainty (Type 2 ). In practice, the model parameters are typically estimated from logs from nearby wells and have unknown uncertainties.

To consider overall uncertainty in the $\mathrm{Xu}$-White model for a fixed set of model parameters, we assume that the estimated seismic velocity and density calculated from reservoir parameters using the $\mathrm{Xu}$-White model are not exact. We model them as a truncated multivariate Gaussian distribution with means determined from the $\mathrm{Xu}$-White model and a covariance matrix determined from an assumed correlation structure and coefficients of variation. Let the variables $V_{P}$, $V_{S}$, and $\rho$ denote seismic $\mathrm{P}$ - and S-wave velocity and density, respectively. Let $\varepsilon_{\mathrm{P}}, \varepsilon_{\mathrm{S}}$, and $\varepsilon_{\rho}$ represent additive errors in the rock-physics model results. Then the conditional probability density function is given by

$$
f\left(V_{p}, V_{s}, \rho \mid S_{w}, \varphi, c, \boldsymbol{\theta}_{2}\right)=\frac{1}{\sqrt{(2 \pi)^{3}|\Sigma|}} \exp \left(-\boldsymbol{\varepsilon}^{T} \Sigma^{-1} \boldsymbol{\varepsilon}\right)
$$

where $\Sigma$ is the covariance matrix, $\boldsymbol{\varepsilon}$ is the vector $\left.{ }_{(\mathrm{P}}, \varepsilon_{\mathrm{S}}, \varepsilon_{\rho}\right)^{T}$, and $c$ represents shale content.

\section{Bayesian model}

The Bayesian model was developed for a layered reservoir. In the reservoir layer, we estimate water saturation $S_{w}$, porosity $\varphi$, and shale content $c$. As in the model given by Chen et al. (2007), we add several layers above and below the reservoir to account for uncertainty in selecting the time window for seismic AVA inversion. For those layers, we invert for elastic bulk and shear moduli (K and $\boldsymbol{\mu})$ and density $\boldsymbol{\rho}_{\mathrm{o}}$, from which we calculate $\mathbf{V}_{P}$ and $\mathbf{V}_{S}$. Because the resistivity $\mathbf{r}_{0}$ of the seawater and the sedimentary overburden also affects the estimates of reservoir parameters, we also consider it as an unknown vector in this model.

Unlike the analysis presented in Chen et al. (2007), we use stochastic rock-physics models in this study. For given reservoir parameters, the calculated reservoir seismic velocities $\mathbf{V}_{P}$ and $\mathbf{V}_{S}$, density $\boldsymbol{\rho}$, and electrical resistivity $\mathbf{r}$ are all considered to be random variables. Let the matrix $\mathbf{R}$ denote seismic AVA data, which are explicit functions of seismic velocity and density within the reservoir, and implicit functions of elastic properties $\left(\mathbf{K}, \boldsymbol{\mu}\right.$, and $\left.\boldsymbol{\rho}_{\mathbf{0}}\right)$ in the zones outside the reservoir. Let the matrix E denote CSEM data, which are functions of reservoir resistivity $\mathbf{r}$ and overburden resistivity $\mathbf{r}_{\mathrm{o}}$. Since CSEM and seismic AVA data are two different types of geophysical measurements, it is reasonable to assume that they are statistically uncorrelated. Therefore, we obtain the following Bayesian model:

$$
\begin{gathered}
f\left(\mathbf{S}_{w}, \mathbf{c}, \boldsymbol{\varphi}, \mathbf{V}_{p}, \mathbf{V}_{s}, \boldsymbol{\rho}, \mathbf{K}, \boldsymbol{\mu}, \boldsymbol{\rho}_{0}, \mathbf{r}, \mathbf{r}_{0}, \boldsymbol{\theta}_{1}, \boldsymbol{\theta}_{2} \mid \mathbf{R}, \mathbf{E}\right) \propto \\
\quad f\left(\mathbf{R} \mid \mathbf{V}_{p}, \mathbf{V}_{s}, \boldsymbol{\rho}, \mathbf{K}, \boldsymbol{\mu}, \boldsymbol{\rho}_{0}\right) f\left(\mathbf{E} \mid \mathbf{r}, \mathbf{r}_{0}\right) \\
\quad f\left(\mathbf{r} \mid \mathbf{S}_{w}, \boldsymbol{\varphi}, \boldsymbol{\theta}_{1}\right) f\left(\mathbf{V}_{p}, \mathbf{V}_{s}, \boldsymbol{\rho} \mid \mathbf{S}_{w}, \boldsymbol{\varphi}, \mathbf{c}, \boldsymbol{\theta}_{2}\right) \\
\quad f\left(\mathbf{S}_{w}, \mathbf{c}, \boldsymbol{\varphi}, \mathbf{K}, \boldsymbol{\mu}, \boldsymbol{\rho}_{0}, \mathbf{r}_{0}, \boldsymbol{\theta}_{1}, \boldsymbol{\theta}_{2}\right)
\end{gathered}
$$

Equation 4 defines a joint a posteriori probability distribution function of all unknown parameters, which is known up to a normalizing constant. The first and second terms on the right side of the equation are the likelihood functions of seismic AVA data and CSEM data, respectively, and are calculated from forward modeling. The third and fourth terms on the right side of the equation are the conditional pdfs given reservoir parameters and parameters associated with the rock physics models; for $\mathbf{r}$, this is given by Equation 2. The final term on the right side of the equation is the a priori distribution of the unknown variables, which we take to have constant pdfs within reasonable bounds.

\section{Synthetic Study}

The synthetic model includes an oil-bearing reservoir embedded in a shale section, lying $1200 \mathrm{~m}$ under the seafloor, with shale content, porosity, and water saturation of $0.1,0.32$, and 0.1 , respectively. Outside the reservoir, the shale content and water saturation in the shale section are constant (100\%), 
whereas the porosity decreases and the background resistivity increases with increasing depth from the seafloor.

The seismic AVA data are NMO-corrected angle gathers generated by convolving a $25 \mathrm{~Hz}$ Ricker wavelet with the angle-dependent reflectivity, which is calculated using the Zoeppritz equations (Aki and Richards, 1980) for each layer interface. The traces are sampled at $2 \mathrm{~ms}$ for seven incidence angles (i.e., 5, 10, 15, 20, 25, 30, and 35 degrees). We assume that the synthetic seismic data include spatially correlated Gaussian random noise and that the spatial correlation is determined by an exponential variogram with an integral length of $12 \mathrm{~ms}$. The variance of the Gaussian noise is angle dependent; the signal-to-noise ratios (SNRs) are 12, 11, 10, 9, 8,7 , and 5 from the near to the far offsets. We use the XuWhite model (Xu and White, 1995) with parameters given in Table 1 to link reservoir parameters to $\mathrm{P}$ - and $\mathrm{S}$-wave velocity and density. (The brine resistivity varies with depth; it is shown for the reservoir level.)

Table 1. Parameters for the Xu-White model and Archie's law

\begin{tabular}{|l|lr|}
\hline Types & Parameters & Values \\
\hline \multirow{5}{*}{ Sand } & Bulk modulus $(\mathrm{GPa})$ & 42.584 \\
& Shear modulus $(\mathrm{GPa})$ & 40.470 \\
& Density $\left(\mathrm{g} / \mathrm{cm}^{3}\right)$ & 2.650 \\
& Aspect ratio & 0.09 \\
\hline \multirow{3}{*}{ Clay } & Bulk modulus $(\mathrm{GPa})$ & 34.260 \\
& Shear modulus $(\mathrm{GPa})$ & 18.504 \\
& Density $\left(\mathrm{g} / \mathrm{cm}^{3}\right)$ & 2.680 \\
& Aspect ratio & 0.06 \\
\hline Brine & Bulk modulus $(\mathrm{GPa})$ & 3.22 \\
& Density $\left(\mathrm{g} / \mathrm{cm}^{3}\right)$ & 1.09 \\
\hline Oil & Bulk modulus $(\mathrm{GPa})$ & 0.75 \\
& Density $\left(\mathrm{g} / \mathrm{cm}^{3}\right)$ & 0.7091 \\
\hline \multirow{3}{*}{ Archie's law } & Porosity exponent & 2.00 \\
& Saturation exponent & 2.00 \\
& Reservoir brine resistivity & 0.11 \\
& $(\Omega-m)$ & \\
\hline
\end{tabular}

The marine CSEM data consist of the electric fields measured at six receivers deployed on the seafloor, with an electric dipole source at five different frequencies $(0.10,0.25$, $0.50,0.75$, and $1.00 \mathrm{~Hz})$. Six source-receiver offsets $(4,5,6$, 7,8 , and $10 \mathrm{~km}$ ) are used. The relationship between electrical resistivity and water saturation and porosity is given by Archie's law using the parameters listed in Table 1. We added $2 \%$ to $4 \%$ relative noise to the synthetic data, with higher noise levels at the farther offsets.

Inversion using rock-physics models with model output uncertainty

We first focus on studying the effect of inherent uncertainty (i.e., on computed $\mathrm{V}_{\mathrm{P}}, \mathrm{V}_{\mathrm{S}}$, etc.) in the rock-physics model
(Type 1) on estimates of reservoir parameters. We invert the synthetic AVA and CSEM data (containing noise) using rock-physics models with uncertainty equivalent to coefficients of variation $(\mathrm{CV})$ of $1 \%, 3 \%, 5 \%$, and $10 \%$.

Figure 1 shows the estimated probability density functions (pdfs) of water saturation, shale content, and porosity using the stochastic rock-physics models. It is evident that uncertainty in the rock-physics models has significant effects on the estimates of reservoir parameters. With an uncertainty of $5 \%$ or more in the rock-physics models, even with our low-noise CSEM data, we cannot estimate shale content. For porosity, the estimates also become poorer with increasing uncertainty in the rock-physics models. Compared to shale content and porosity, the water saturation estimate is less sensitive to uncertainty in the rock-physics models.
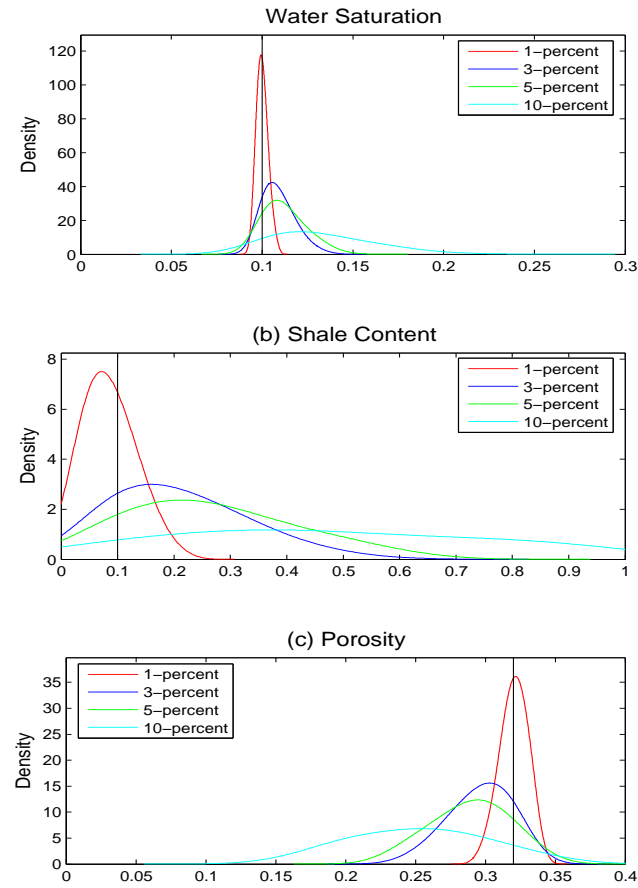

Figure 1. The estimated pdfs of water saturation, shale content, and porosity when the rock-physics models have the specified levels of overall uncertainty.

Inversion using rock-physics models with both model and parameter uncertainty

Parameter uncertainty (Type 2) in rock-physics models (e.g., in the Archie's Law exponents $m$ and $n$ ) is often ignored because of the difficulty of incorporating it in the estimation. 
The Bayesian model developed in this study provides a flexible, integrated approach for investigating all sources of uncertainty simultaneously. To investigate the effects of parameter uncertainty, we assume that each model parameter lies in a given range and that the probability of finding any value within that range is constant. In the following, we consider four levels of uncertainty: $1 \%, 3 \%, 5 \%$, and $10 \%$ around the corresponding true values of the model parameters, while we keep the overall uncertainty in the rockphysics model outputs constant (at $1 \%$ ).

Figure 2 shows the estimated pdfs of water saturation, shale content, and porosity for this case. For our low-noise CSEM data (i.e., relative errors between $2 \%$ and $4 \%$ ) and small inherent errors in the rock-physics models $(1 \%)$, the estimated reservoir parameters still have unsatisfactorily large errors when the uncertainty in the rock-physics model parameters is $5 \%$ or more. Note that water saturation is again the most robustly estimated quantity.

\section{Conclusions}

The Bayesian model that we have developed provides a unified and conceptually consistent approach for analyzing various uncertainties in reservoir parameter estimation, such as measurement errors, model uncertainties, and parameter uncertainties. The synthetic study shows that uncertainty in both rock-physics models and in their associated parameters can, as expected, have significant effects on reservoir parameter estimation, especially when those models and their associated parameters are subject to errors of several percent or more. Without considering uncertainty in rock-physics models, we may be overly optimistic about the precision of our estimates of reservoir parameters. The framework presented here also provides a method for estimating the impact of other sources of uncertainty on inversion results.

\section{Acknowledgments}

We thank ExxonMobil Upstream Research Company for providing funding for this study and for permission to publish this work. We also thank Shiyu Xu and Chris Finn for many useful discussions.

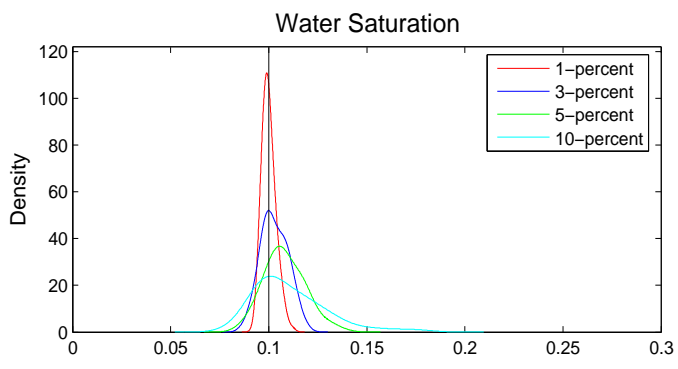

(b) Shale Content

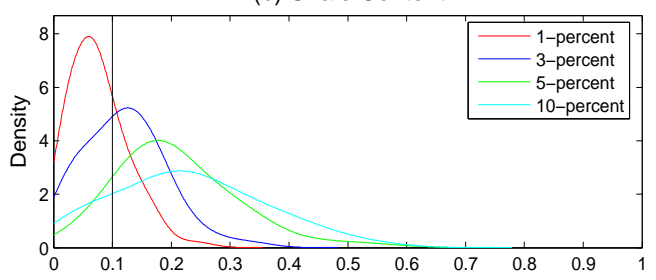

(c) Porosity

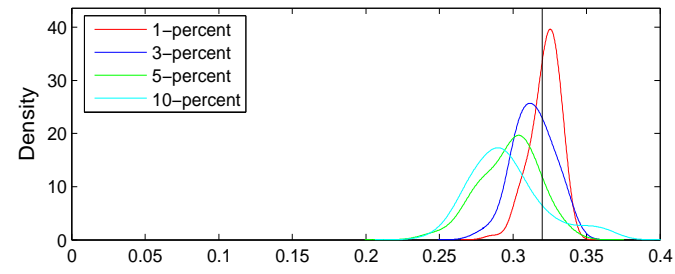

Figure 2. The estimated pdfs of water saturation, shale content, and porosity when the parameters associated with the stochastic rock-physics models have the specified levels of uncertainty; the overall model uncertainty is set to $1 \%$ for each case. Comparison with Figure 1 shows that a given level of uncertainty in the model parameters has a smaller effect than the same level of uncertainty in the model result. 